\title{
Drosophila tao Controls Mushroom Body Development and Ethanol-Stimulated Behavior through par-1
}

\author{
Ian King, ${ }^{1}$ Linus T.-Y. Tsai, ${ }^{1}$ Ralf Pflanz, ${ }^{3}$ Aaron Voigt, ${ }^{3}$ Seongsoo Lee, ${ }^{4}$ Herbert Jäckle, ${ }^{3}$ Bingwei Lu, ${ }^{4}$ \\ and Ulrike Heberlein ${ }^{1,2}$ \\ ${ }^{1}$ Department of Anatomy, University of California, San Francisco, San Francisco, California 94143, 2Ernest Gallo Clinic and Research Center, Emeryville, \\ California 94608, ${ }^{3}$ Max Planck Institute for Biophysical Chemistry, D-37077 Göttingen, Germany, and ${ }^{4}$ Department of Pathology, Stanford University \\ Medical Center, Stanford, California 94305-5324
}

In both mammalian and insect models of ethanol-induced behavior, low doses of ethanol stimulate locomotion. However, the mechanisms of the stimulant effects of ethanol on the CNS are mostly unknown. We have identified tao, encoding a serine-threonine kinase of the Ste20 family, as a gene necessary for ethanol-induced locomotor hyperactivity in Drosophila. Mutations in tao also affect behavioral responses to cocaine and nicotine, making flies resistant to the effects of both drugs. We show that tao function is required during the development of the adult nervous system and that tao mutations cause defects in the development of central brain structures, including the mushroom body. Silencing of a subset of mushroom body neurons is sufficient to reduce ethanol-induced hyperactivity, revealing the mushroom body as an important locus mediating the stimulant effects of ethanol. We also show that mutations in par-1 suppress both the mushroom body morphology and behavioral phenotypes of tao mutations and that the phosphorylation state of the microtubule-binding protein Tau can be altered by RNA interference knockdown of tao, suggesting that tao and par-1 act in a pathway to control microtubule dynamics during neural development.

\section{Introduction}

Drosophila are highly adapted to an environment in which they frequently encounter ethanol throughout their life cycle (Guarnieri and Heberlein, 2003). Ethanol has behavioral effects in flies that parallel those seen in mammals, including preference for ethanol consumption (Devineni and Heberlein, 2009), induction of tolerance (Scholz et al., 2000), sedation at high ethanol concentrations (Moore et al., 1998), and stimulation of locomotor activity at low to moderate concentrations (Wolf et al., 2002). Characterizing the mechanisms by which ethanol-induced behaviors occur in Drosophila will thus shed light on how Drosophila has evolved to deal with an important ethological stimulus and will also provide a model for the effects of ethanol on more complex animals, including humans.

In both mammals and in Drosophila, ethanol-induced locomotor activity is thought to be a correlate of the disinhibiting and rewarding effects of ethanol (Phillips and Shen, 1996; Wolf et al.,

Received Aug. 20, 2010; revised 0ct. 28, 2010; accepted Nov. 12, 2010.

This work was supported by National Institute on Drug Abuse Grant 5F32DA19731-3 (I.K.) and National Institute on Alcohol Abuse and Alcoholism Grants AA10035 and AA017072-component 7 (U.H.), and by National Institute of Mental Health Grant MH080378 and McKnight Foundation Brain Disorders Award (B.L.). Fly strains were provided by Denise Montell, Barry Dickson, Kimihiro Sato, Yuh-Nung Jan, and the Bloomington and Kyoto Stock Centers. Antibodies were from the Developmental Studies Hybridoma Bank. We thank Tina Schwabe for assistance with pupal brain dissections and Yuh-Nung Jan for use of equipment and assistance with confocal microscopy.

Correspondence should be addressed to Ulrike Heberlein at the above address. E-mail: ulrike.heberlein@ucsf.edu.

I. King's present address: Department of Cell and Molecular Physiology, University of North Carolina, Chapel Hill, NC 27599-7545.

L. T.-Y. Tsai's present address: Beth Israel Deaconness Hospital, Harvard University, Boston, MA 02215-5491.

A. Voigt's present address: University Medical Center, Rheinisch-Westfälische Technische Hochschule Aachen, Pauwelsstrasse 30, D-52074 Aachen, Germany.

DOI:10.1523/JNEUROSCI.4416-10.2011

Copyright $\odot 2011$ the authors $\quad 0270-6474 / 11 / 311139-10 \$ 15.00 / 0$
2002). In Drosophila, as in mammals, ethanol-induced enhancement of locomotor activity is mediated by the dopaminergic system (Phillips and Shen, 1996; Bainton et al., 2000; Wolf et al., 2002), in conjunction with neurons of the ellipsoid body. However, the neural circuitry mediating the locomotor stimulant effects of ethanol in flies has not been defined in any greater detail.

tao (CG14217) is the single representative in Drosophila of the TAO kinase subfamily of Ste20 serine-threonine kinases (Dan et al., 2001). Drosophila Tao is highly similar to all three mammalian TAO family proteins, $\mathrm{TAO} 1, \mathrm{TAO} 2$, and $\mathrm{TAO} 3$ (60-70\% identity in the kinase domain and $45 \%$ identity in the conserved C-terminal domain). Mammalian TAO1 is part of a signaling pathway that can control the extension of neurites in immature cultured neurons through its effects on the microtubule cytoskeleton. TAO1 can phosphorylate and activate the kinase Par-1, which in turn phosphorylates the mictotubule-stabilizing protein Tau, reducing its affinity for microtubules (Biernat et al., 2002; Timm et al., 2003, 2006) and promoting the extension of microtubule-supported processes.

We have identified a mutation, $t a 0^{E P 1455}$, that causes resistance to the locomotor stimulant effects of ethanol. We found that $t a 0^{E P 1455}$ mutant flies have defects in the morphology of the central brain, including the mushroom body (MB). Silencing subsets of $\mathrm{MB}$ neurons reduces ethanol-induced hyperactivity, identifying these neurons as part of the circuitry that mediates the stimulant effects of ethanol. We also show both that the behavioral and morphological phenotypes of $t a 0^{E P 1455}$ can be suppressed by mutations in par-1 and that tao mutations can increase Par-1-induced Tau phosphorylation. These results suggest that tao negatively regulates par-1 as part of a signaling pathway in 
developing neurons that controls Tau phosphorylation and that this pathway controls development of $\mathrm{MB}$ neurons required for ethanol-induced hyperactivity.

\section{Materials and Methods}

Generation and mapping of the tao ${ }^{50}$ deletion. To generate a loss-offunction allele of tao, the EP transposon in $t a o^{E P 1455}$ was mobilized by crossing to a line carrying $\Delta 2-3$ transposase. Revertant lines were recovered by screening for loss of the mini-white marker and checked for lethality. The $t a 0^{50}$ lethal mutation was mapped by amplification of the genomic region surrounding the $t a o^{E P 1455}$ insertion site by PCR with a primer set bracketing the tao transcription start site. PCR products were subsequently cloned and sequenced to determine the break points of the deletion. Sequencing revealed a 2077 bp deletion from 753 bp upstream to $1324 \mathrm{bp}$ downstream of the transcription start site for the D and $\mathrm{E}$ transcripts of tao (which corresponds to base pair 19,473,841 of Flybase release FB2010_06 from June 2010 www.flybase.org). The deletion uncovers all of exon 1 of transcripts D and E. In addition to the major deletion, tao50 contains four smaller upstream deletions, at the following positions upstream relative to the tao transcription start site: 2301-2313 (12 bp), 2294-2299 (5 bp), 902-905 (3 bp), and 882-900 (18 bp).

Construction of tao genomic fragment transgenic line. BAC clone BACR10m08 (Hoskins et al., 2000) was digested with Pfl23II, a 33,686 bp fragment isolated and further digested using Not1 and Xba1. A $19,971 \mathrm{bp}$ fragment was isolated and cloned into corresponding sites in pPTGal (Sharma et al., 2002) to generate genomic fragment NX19, uncovering the exon coding and $6.8 \mathrm{~kb}$ of upstream and $4.5 \mathrm{~kb}$ of downstream genomic sequence. Transgenic flies were generated by pole plasm injection and P-element-mediated genomic integration (Rubin and Spradling, 1982).

Tao expression and rabbit immunization. A 1068 bp BamH1-Sac1 fragment from LD40388 was inserted in corresponding sites of pRSet_B to produce a 398 aa $6 \times$ His tagged fusion protein containing 357 aa (amino acids 10-366) from the kinase domain of Tao (long). Expression was induced in BL1-CodonPlus cells (Stratagene) and fusion protein was purified using Protino Ni-NTA columns (Macherey-Nagel). Rabbits were immunized by Eurogentec using "classic" conditions (three boosts in 3 months).

Behavioral assays and analysis. All flies assayed for behavior were backcrossed for at least five generations to the $w^{1118}$ Berlin genetic background, unless otherwise noted. Assays for behavioral response to cocaine and nicotine were performed as described previously (Bainton et al., 2000). Assays for ethanol-induced hyperactivity and sedation were performed as described previously (Wolf et al., 2002; Kong et al., 2010). Ethanol hyperactivity assays used a concentration of $33 \%$ ethanol vapor (50:100 arbitrary flow units), except where noted. For standard analysis, $21 \mathrm{~min}$ movies generated in Adobe Premier were analyzed at 14 time points using a modified version of DIAS tracking software. For detailed startle analysis, $4 \mathrm{~min}$ movies were analyzed at 16 time points. Startle assays also used a concentration of $33 \%$ ethanol vapor.

To more closely synchronize ages in temperature shift experiments, fly cultures were generated using 40 females laying eggs for $24 \mathrm{~h}$, rather than 20 females for $48 \mathrm{~h}$ as in other experiments. All assays represent $n>7$, with experiments performed on at least three separate days, from at least two independent sets of crosses.

Statistical analysis. Distance traveled during hyperactivity assays was estimated by summing average traces for time points between 7 and 20 min, as described previously (Kong et al., 2010). Summed traces were compared by one-way ANOVA, with Bonferroni's correction for multiple comparisons where appropriate. Where multiple comparisons were made, experiments were compared with all controls. Values of $p$, where indicated, hold for all comparisons of experiment to controls. Error bars represent SEM.

Ethanol absorption assays. Ethanol content of flies was measured using a colorimetric enzymatic assay kit. Groups of 30 flies were exposed to nonsedating doses of ethanol (47\%) for up to $30 \mathrm{~min}$. Flies were flash frozen in liquid nitrogen at the indicated time point, and then assayed for ethanol content.
Western blotting. Western blots were performed using standard procedures. Primary antibodies against Tao and $\alpha$-tubulin (mouse monoclonal B-5-1-2) were diluted 1:20,000 in 5\% milk. Anti-phospho-Tau (mouse monoclonal 12E8) was diluted 1:2000. Anti-Tau (mouse monoclonal T14) was diluted 1:2000. HRP-conjugated anti-mouse IGG and anti-rabbit IGG secondary antibodies were diluted 1:10,000 in 5\% milk. Blots were visualized using ECL Plus and Hyperfilm (GE Healthcare).

Immunohistochemistry. Whole-mount brains were dissected in PBS, fixed for $30 \mathrm{~min}$ at room temperature in PBS with $4 \%$ formaldehyde, washed twice in PBS with $0.3 \%$ Triton X-100 (PBT 0.3), and blocked in PBT 0.3 with $5 \%$ normal goat serum. Samples were incubated with primary antibody overnight, diluted as follows in PBT 0.3 with 5\% normal goat serum: NC82 (anti-bruchpilot), 1:500; anti-Fasciclin II, 1:200. After treatment with primary antibody, samples were washed three times in PBT 0.3 and incubated overnight with fluorescently labeled secondary antibody diluted 1:500. Samples were again washed three times in PBT 0.3 before mounting.

\section{Results}

tao $^{E P 1455}$ shows impaired ethanol-induced hyperactivity

We screened a collection of P-element insertion lines (Rørth, 1996) for strains with altered responses to drugs of abuse and identified the line $\mathrm{EP}(\mathrm{X}) 1455$, a P-element insertion in the $\mathrm{X}$-linked gene tao, as a mutant with dramatically altered response to ethanol. We examined the behavior of male flies at a moderate, nonsedating concentration of ethanol, at which wild-type flies exhibit strong locomotor hyperactivity but not ethanol-induced sedation. At this concentration, $t a o^{E P 1455}$ flies were dramatically less hyperactive than the control strain (Fig. $1 A, B$ ). tao $^{E P 1455}$ also displayed reduced hyperactivity over a range of ethanol doses (supplemental Fig. S1C, available at www.jneurosci.org as supplemental material), showing that this mutation does not simply shift the flies' dose-response to ethanol but instead prevents flies from mounting a normal hyperactivity response to ethanol at any concentration.

We performed secondary tests to determine whether this behavioral phenotype of $\operatorname{tao}^{E P 1455}$ flies was attributable simply to a defect in locomotion. Flies have a strong tendency for negative geotaxis and positive phototaxis: when placed in a cylinder with a light source above, wild-type flies will climb quickly to the top. To test the locomotor ability of $\operatorname{tao}^{E P 1455}$, we placed single flies in a cylinder and measured their speed of climbing in straight-line bouts of at least $50 \mathrm{~mm}$ (Bland et al., 2009). The average walking speed of $t a o^{E P 1455}$ flies was equal to that of wild-type flies, indicating that $t a o^{E P 1455}$ flies are capable of normal locomotion (supplemental Fig. S1 A, available at www.jneurosci.org as supplemental material). As another test of locomotion, we examined in detail the startle phase of the ethanol response. Naive flies go through a brief burst of enhanced locomotion in response to the smell of ethanol vapor (Wolf et al., 2002). $t a o^{E P 1455}$ flies startled normally, reaching speeds that are usually observed in the later phases of the hyperactivity response to ethanol (Fig. 1C). This analysis shows that $t a o^{E P 1455}$ flies can smell ethanol and apparently do not have significantly impaired sensory or motor systems.

We also measured the rate at which $\operatorname{tao}^{E P 1455}$ flies accumulated ethanol over the time course of our behavioral assay. $t a o^{E P 1455}$ flies accumulated ethanol at the same rate as wild-type flies, indicating that the phenotype of the tao ${ }^{E P 1455}$ line is not attributable to altered ethanol absorption kinetics (supplemental Fig. S1B, available at www.jneurosci.org as supplemental material).

To determine whether tao ${ }^{E P 1455}$ impairs responses to ethanol specifically, we tested the sensitivity of tao ${ }^{E P 1455}$ flies to other drugs of abuse. When exposed to volatilized nicotine or freebase cocaine, flies fail to exhibit normal negative geotaxis. A drug ef- 
A

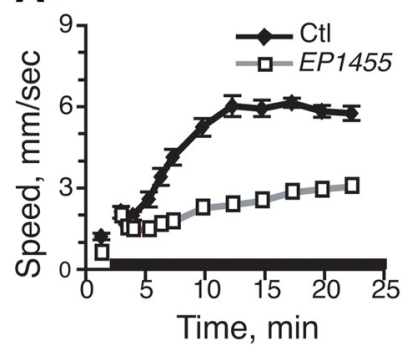

B

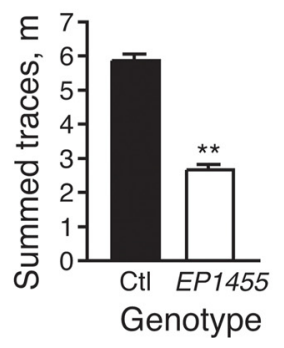

C

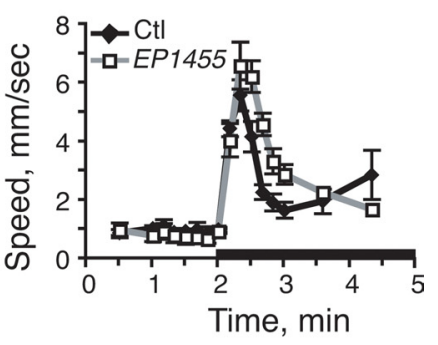

Figure 1. $\quad t a 0^{E P 1455}$ displays reduced ethanol-induced hyperactivity. $\boldsymbol{A}$, Groups of wild-type and $t a 0^{E P 1455}$ flies were exposed to a moderate, nonsedating concentration of ethanol vapor (33\%) for $21 \mathrm{~min}$. Ethanol exposure begins at $t=2 \mathrm{~min}$, indicated by the black horizontal bar. The average speed of the population was determined using DIAS image analysis software at intervals throughout the course of the assay. $\boldsymbol{B}$, Quantification of ethanol-induced hyperactivity. An estimate of the average total distance traveled during the assay was obtained by summing average traces from 7.5, 10,12.5, 15, 17.5, 20, and 22.5 min time points and multiplying by time (Kong et al., 2010). C, Detailed analysis of olfactory startle. Flies were exposed to ethanol vapor as in $\boldsymbol{A}$, but movies were analyzed in detail over a short time course. Ethanol is administered at $t=2 \mathrm{~min}$. Genotypes were compared by one-way ANOVA. ${ }^{* *} p<0.01$. Error bars represent SEM. See also supplemental Figure S1 (available at www.jneurosci.org as supplemental material).
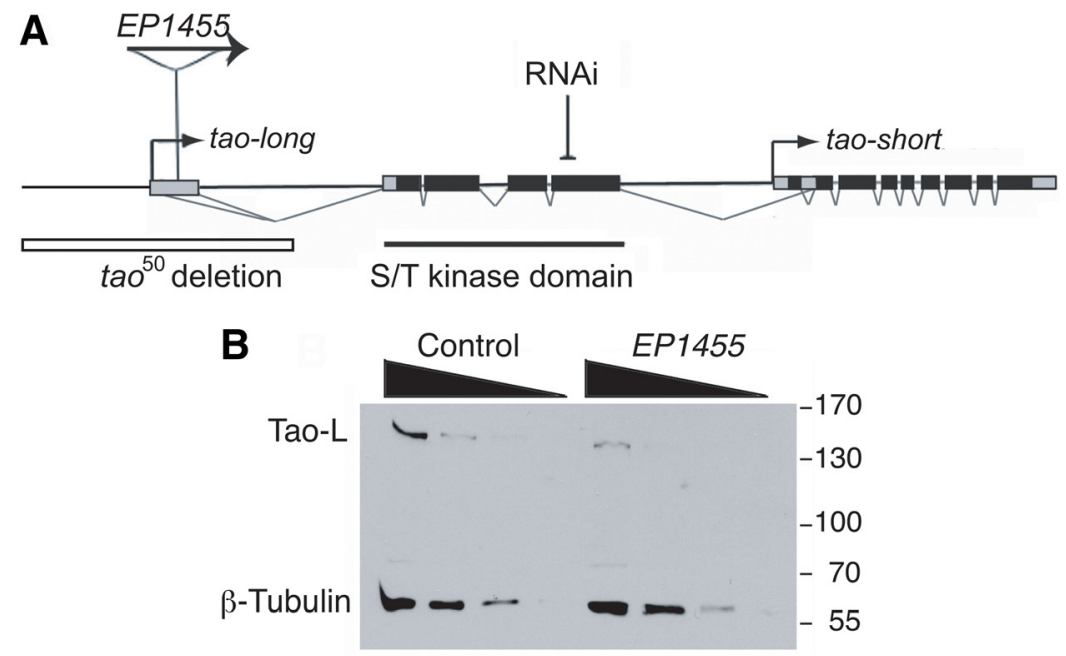

C

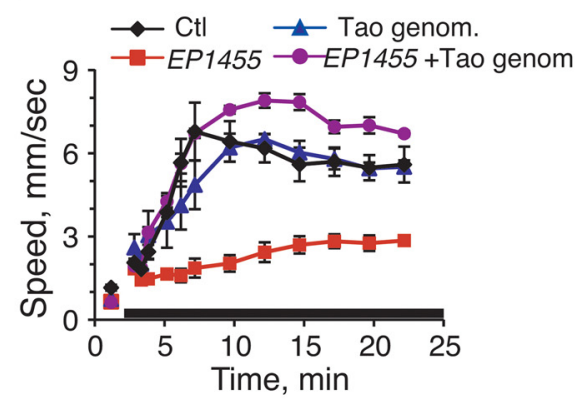

D

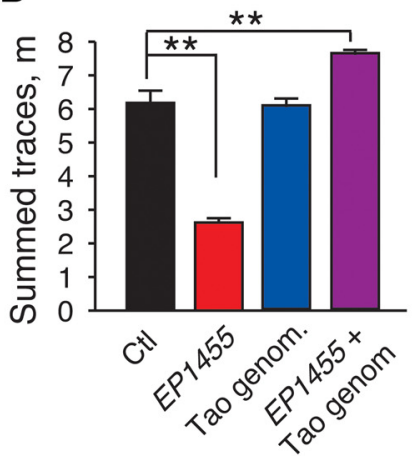

Figure 2. Reduced Tao expression is responsible for the impaired ethanol-induced hyperactivity seen in EP1455 flies. $A$, Schematic of the tao gene. Location of the $t a 0^{E P 1455}$ insert in the $5^{\prime}$-UTR is indicated, with orientation of the EP promoter indicated by the arrow. Transcription start sites for long and short forms of Tao are indicated with arrows. The location of the inverted-repeat sequence used to generate RNAi knockdown and the location of sequences deleted in ta050 are also indicated. Translated regions are indicated in black, and untranslated regions, in gray. $\boldsymbol{B}$. Western blot of total protein from wild-type and tao ${ }^{E P 1455}$ male flies, probed with anti-Tao and anti- $\alpha$-tubulin. Size markers are indicated to the right of the blot. For each genotype, a serial dilution of protein from $0.5,0.25$, and 0.125 flies was loaded. C, Ethanol-induced hyperactivity of flies carrying the $t a 0^{E P 1455}$ mutation along with a transgenic genomic fragment of $19 \mathrm{~kb}$ containing tao. D, Quantification of summed traces from C. Genotypes were compared by one-way ANOVA with Bonferroni's correction for multiple comparisons. ${ }^{* *} p<0.01$. Error bars represent SEM. See also supplemental Figure S2 (available at www.jneurosci.org as supplemental material).

fect score for cocaine or nicotine can be calculated by determining the proportion of a group of flies that fails to climb after drug exposure (Bainton et al., 2000). We exposed male wild-type and tao ${ }^{E P 1455}$ flies to cocaine and nicotine and assayed drug effects in this negative geotaxis assay. tao ${ }^{E P 1455}$ flies were greatly resistant to the effects of both cocaine and nicotine (supplemental Fig. S1D, available at www. jneurosci.org as supplemental material). It appears, then, that $t a 0^{E P 1455}$ does not cause an impairment of the response to ethanol specifically but instead alters acute sensitivity to multiple drugs of abuse. The fact that $t a 0^{E P 1455}$ flies show resistance to cocaine and nicotine (i.e., they climb better than control flies when exposed to the drugs) is additional evidence that $t a o^{E P 1455}$ flies are not generally weak and defective in locomotion.

Together, these experiments show that $t a 0^{E P 1455}$ drastically disrupts normal ethanol-induced hyperactivity, as well as reducing the response to cocaine and nicotine. The ethanol phenotype is not simply attributable abnormal ethanol pharmacokinetics or a locomotor impairment but instead reflects a defect in higher-level control of drug-induced behavior.

\section{tao is required in neurons for} ethanol-induced hyperactivity tao ${ }^{E P 1455}$ is an insertion in the $5^{\prime}$-untranslated region (UTR) of tao. Figure $2 A$ shows a schematic of the tao locus; the tao gene is predicted to produce four transcripts that encode two distinct proteins. The long form of Tao is predicted to contain both an N-terminal kinase domain and a conserved C-terminal domain found in all TAO family kinases. The short form is predicted to contain only the C-terminal domain. A previous study found that a transcript of the length predicted to encode for the short form is expressed during early development but does not persist beyond late embryonic stages; transcripts encoding the long form are expressed throughout the life of the fly (Sato et al., 2007).

To exclude the possibility that a secondsite mutation was responsible for the phenotype of $t a 0^{E P 1455}$, we isolated lines with precise excision of the P-element. In these strains, ethanol-induced hyperactivity was completely restored (supplemental Fig. $\mathrm{S} 2 A, B$, available at www.jneurosci.org as supplemental material), indicating that the P-element is directly responsible for the mutant phenotype. To establish whether the EP1455 element reduces Tao protein expression, we probed Western blots of total 
protein from adult flies with a polyclonal antibody raised to the tao kinase domain. Indeed, we found that $t a o^{E P 1455}$ impairs Tao expression: in hemizygous mutant males, expression of Tao protein was reduced by $\sim 50 \%$ (Fig. $2 B$ ).

To verify that the mutant phenotype of tao ${ }^{E P 1455}$ is caused by reduced Tao expression, we introduced a normal copy of tao into the mutant using a genomic transgene. Recombinant flies carrying both tao ${ }^{E P 1455}$ and a transgenic genomic copy of tao exhibited normal, or slightly elevated, hyperactivity (Fig. 2C,D), showing that restoration of Tao expression could rescue the behavioral phenotype of tao $^{E P 1455}$. We also observed that tao ${ }^{E P 1455}$ failed to complement $t a o^{50}$, a deletion allele of tao that eliminates most or all Tao expression (supplemental Fig. S2C, available at www.jneurosci.org as supplemental material). Females heterozygous for either $t a o^{E P 1455}$ or $t a o^{50}$ hyperactivated normally in response to a moderate concentration of ethanol, but $\operatorname{tao}^{E P 1455} / \mathrm{tao}^{50}$ flies had greatly diminished hyperactivity (supplemental Fig. S2D,E, available at www.jneurosci.org as supplemental material). We conclude that the P-element insertion in $\operatorname{tao}^{E P 1455}$ reduces Tao expression and that lowered Tao levels are responsible for the mutant's dramatic effects on ethanol-induced hyperactivity.

To determine whether Tao function is required in neurons for normal ethanol-induced hyperactivity, we expressed Tao specifically in neurons in the tao ${ }^{E P 1455}$ mutant background. The EP transposon contains a UAS element and core promoter that allow GAL4-driven expression of downstream sequences (Rørth, 1996), and the tao ${ }^{E P 1455}$ insertion is oriented to allow GAL4-dependent expression of Tao (Fig. 2A) (Shulman and Feany, 2003). When tao ${ }^{E P 1455}$ was combined with elav-GAL4, which drives GAL4 expression in all neurons (Lin and Goodman, 1994), the flies' ethanol-induced hyperactivity was restored to normal levels (Fig. $3 A, B$ ), indicating that neuronal-specific expression of Tao is sufficient to rescue the behavioral phenotype of the tao ${ }^{E P 1455}$ mutant.

To test the requirement for Tao function in neurons, we also knocked down tao expression specifically in neurons using RNA interference (RNAi). A transgene expressing a double-stranded RNA targeting a sequence in the kinase domain of tao (UAStao ${ }^{R N A i}$ ) (Fig. $2 A$ ) was combined with elav-GAL4 to silence tao expression in neurons. tao RNAi knockdown sharply reduced levels of Tao in adult head extracts, as seen in Western blots probed with anti-Tao (Fig. 3C). Compared with controls, tao knockdown flies had reduced ethanol-induced hyperactivity (Fig. $3 D, E$ ). This demonstrates that the effects of tao mutants on ethanol-induced hyperactivity are attributable to a reduction in Tao expression in neurons.

tao function is required during metamorphosis for normal ethanol responsiveness and for proper central brain development

In the course of our experiments, we observed that the behavioral phenotype of $t a o^{E P 1455}$ was temperature sensitive. $t_{a o^{E P 1455}}$ flies
B

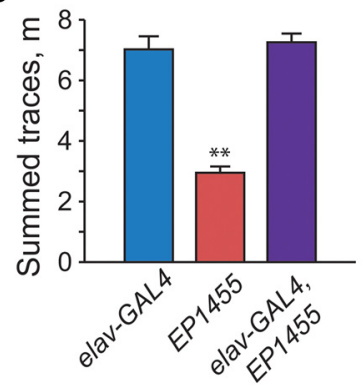

E

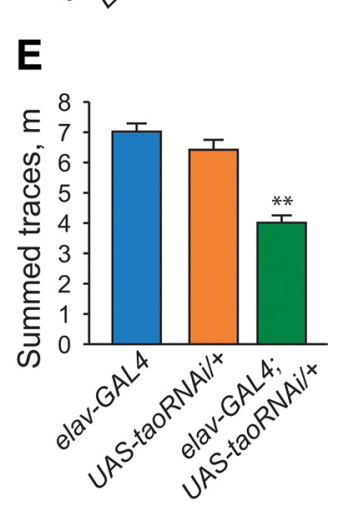

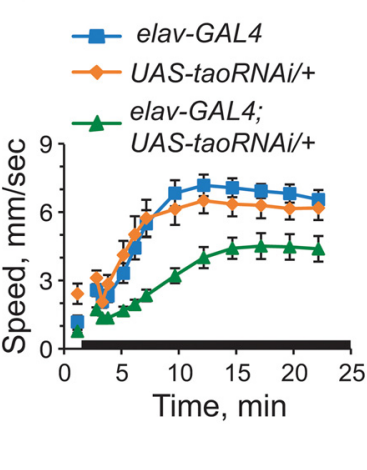

D

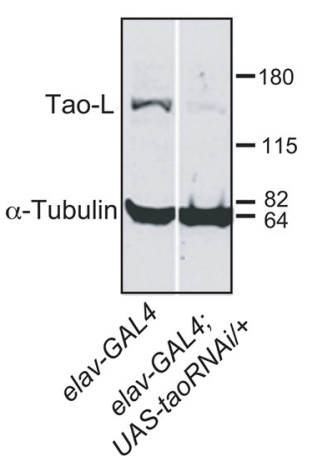

Figure 3. tao function is required in neurons for ethanol-induced hyperactivity. $\boldsymbol{A}$, Ethanol-induced hyperactivity of flies with RNAi knockdown of tao. $\boldsymbol{E}$, Quantification of summed traces from $\boldsymbol{D}$. Genotypes were compared by one-way ANOVA with Bonferroni's correction for multiple comparisons. ${ }^{* *} p<0.01$. Error bars represent SEM.

raised at $25^{\circ} \mathrm{C}$ displayed reduced ethanol-induced hyperactivity (Fig. $4 A, B$ ), whereas $t a o^{E P 1455}$ flies raised at $18^{\circ} \mathrm{C}$ throughout their development showed normal ethanol-induced hyperactivity (Fig. $4 C, D$ ). To define the temperature-sensitive period, we raised flies using varying temperature shift regimens. We found that the phenotype of $t a o^{E P 1455}$ was dependent on the temperature during metamorphosis. $\operatorname{tao}^{E P 1455}$ flies that were raised at $25^{\circ} \mathrm{C}$, shifted to $18^{\circ} \mathrm{C}$ before pupariation, and returned to $25^{\circ} \mathrm{C}$ after eclosion, displayed normal ethanol-induced hyperactivity (Fig. $4 E, F$ ). Conversely, tao $^{E P 1455}$ flies raised at $18^{\circ} \mathrm{C}$, then shifted to $25^{\circ} \mathrm{C}$ to undergo metamorphosis, and returned to $18^{\circ} \mathrm{C}$ after eclosion, displayed the reduced-hyperactivity phenotype of the mutant (Fig. 4G,H). From these experiments, we conclude that tao function is most likely required during metamorphosis for ethanol-induced hyperactivity.

Because the critical period for temperature sensitivity of tao ${ }^{E P 1455}$ occurs during metamorphosis, when the Drosophila nervous system is extensively remodeled to construct the adult nervous system (Truman, 1990), we hypothesized that tao ${ }^{E P 1455}$ might cause defects in the formation of the neural circuits that mediate a normal response to ethanol. We compared the morphology of adult brains from $t a o^{E P 1455}$ with wild-type brains using NC82 staining to define the major neuropil regions of the central brain. Although the brains of tao ${ }^{E P 1455}$ flies were normal in size and in gross morphology, we discovered striking differences in two well defined neuropil structures, the $\mathrm{MB}$ and the ellipsoid body (EB) (supplemental Fig. S3A-J, available at www.jneurosci.org as supplemental material). Most components of the central complex appeared to be unaffected in tao ${ }^{E P 1455}$ brains, including the fan-shaped body and proto- 

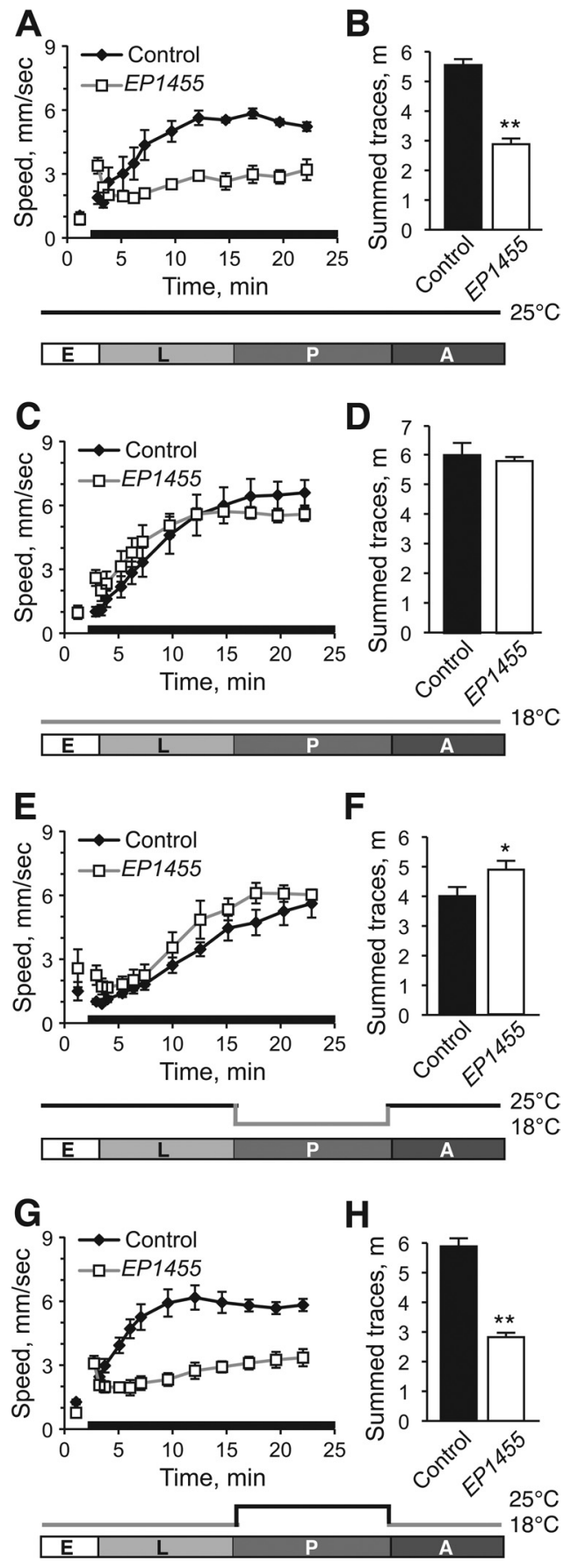

Figure 4. Temporal mapping of $t a 0^{E P 1455}$ temperature sensitivity. $\boldsymbol{A}-\boldsymbol{D}$, Mutant and wildtype flies raised at $25^{\circ} \mathrm{C}(\boldsymbol{A}, \boldsymbol{B})$ and $18^{\circ} \mathrm{C}(\boldsymbol{C}, \boldsymbol{D})$ were tested for ethanol-induced hyperactivity. $\boldsymbol{E}$, $\boldsymbol{F}$, Flies raised at $25^{\circ} \mathrm{C}$ during the embryonic and larval stages were shifted to $18^{\circ} \mathrm{C}$ before pupation, and then returned to $25^{\circ} \mathrm{C}$ for $2 \mathrm{~d}$ after eclosion. $\mathbf{G}, \boldsymbol{H}$, Flies raised at $18^{\circ} \mathrm{C}$ were shifted to $25^{\circ} \mathrm{C}$ before pupation, and then returned to $18^{\circ} \mathrm{C}$ for $2 \mathrm{~d}$ after eclosion. E, Embryo; L, larva; $\mathrm{P}$, pupa; $A$, adult. Genotypes were compared by one-way ANOVA. ${ }^{*} p<0.05 ;{ }^{* *} p<0.01$. Error bars represent SEM.

cerebral bridge (supplemental Fig. S3C,H, available at www. jneurosci.org as supplemental material) (data not shown). However, the EB, which is normally circular, appeared open and flattened in the brains of $t a o^{E P 1455}$ flies (supplemental Fig. $\mathrm{S} 3 B, C, G, H$, available at www.jneurosci.org as supplemental material). We also observed defects in the axonal projections, or lobes of the MB. In wild-type flies, the medial and dorsal lobes of the MB were visible by immunostaining with NC82, but in $t_{a} o^{E P 1455}$ flies they were absent (supplemental Fig. $\mathrm{S} 3 A, B, F, G$, available at www.jneurosci.org as supplemental material).

Staining with an antibody to Fasciclin II (FasII), which labels neurons of the EB as well as $\mathrm{MB} \alpha / \beta$ and $\gamma$ lobes, confirmed the abnormal structure of the EB in $t a o^{E P 1455}$ flies (Fig. $5 A, B$ ). FasII staining also revealed that $t a o^{E P 1455}$ flies mostly lacked MB $\alpha / \beta$ lobes (Fig. $5 A, B) . \gamma$ lobes were present in tao ${ }^{E P 1455}$ brains but were often thinner than in wild-type flies. A striking feature of tao ${ }^{E P 1455}$ brains was a ball-like accumulation of FasII staining in the posterior of the brain, adjacent to the $\mathrm{MB}$ calices (Fig. $5 C, D$ ). This feature was also visible with NC82 staining (supplemental Fig. S3 E, J, available at www.jneurosci.org as supplemental material). Strong RNAi knockdown of tao with the MB-specific driver OK107-GAL4 also caused this phenotype (Fig. 5E-J), and the ball-like structure was only present when MB lobes were absent. This characteristic "ball" phenotype is very similar to a phenotype described for mutations in Rac family small GTPases, which results from an axon guidance defect ( $\mathrm{Ng}$ et al., 2002). For this reason, we believe that tao mutations cause a defect in axon guidance in developing $\mathrm{MB}$ neurons, resulting in adult flies lacking MB lobes.

Since anti-FasII does not stain $\mathrm{MB} \alpha^{\prime} / \beta^{\prime}$ lobes, we visualized all MB lobes using OK107-GAL4-driven expression of CD8.GFP (a membrane-bound form of GFP). We combined strong tao RNAi (coexpression of $U A S-$ tao ${ }^{R N A i}$ and $U A S-D c r 2$ ) with expression of CD8-GFP to examine whether the development of $\alpha^{\prime} / \beta^{\prime}$ lobes was also affected by loss of tao function. With strong tao RNAi, we observed defects in MB lobe morphology in all 16 brain hemispheres examined (Fig. $5 E-J$ ). All $\alpha / \beta$ lobes examined were disrupted to some degree, most being shorter or thinner than normal. Some $\alpha^{\prime}$ lobes were fully formed (3 of 16, or 19\%), but no normal $\beta^{\prime}$ lobes were observed. $\gamma$ lobes were present in most brain hemispheres but were often diffuse and shortened. We conclude that tao knockdown affects development of all three classes of MB neurons. However, the phenotype caused by loss of tao was more severe and more penetrant in $\alpha / \beta$ neurons than in $\alpha^{\prime} / \beta^{\prime}$ or $\gamma$ neurons.

To determine whether tao knockdown disrupts brain development during metamorphosis-the developmental period during which tao function was required for normal behavioral response to ethanol-we examined $\mathrm{MB}$ morphology before and after pupariation in flies in which CD8.GFP and strong tao RNAi expression was driven with OK107-GAL4. The MB of third-instar larvae consist mainly of $\gamma$ and $\alpha^{\prime} / \beta^{\prime}$ neurons (with $\gamma$ neurons having both medial and dorsal projections), whereas $\alpha / \beta$ neurons are added after pupariation (Lee et al., 1999). Third-instar tao knockdown brains showed no apparent defects in MB morphology, having intact dorsal and medial lobes (supplemental Fig. S3 $K, L$, available at www.jneurosci.org as supplemental material). However, $24 \mathrm{~h}$ after pupariation, when MB lobes consist of all three classes of neurons, defects were apparent in the dorsal lobes, which were greatly reduced or absent in tao knockdown animals (supplemental Fig. S3 $M, N$, available at www.jneurosci. org as supplemental material). Thus, the developmental period in which morphological defects develop in tao knockdown animals corresponded to the temperature-sensitive period of the tao ${ }^{E P 1455}$ behavioral phenotype, further suggesting that the reduced ethanol-induced hyperactivity of tao mutants is attributable to abnormal development of central brain circuits that control this behavior. 
Mushroom body neurons mediate ethanol-induced hyperactivity

Because the axon projections of $\mathrm{MB}$ neurons are clearly disrupted in the tao ${ }^{E P 1455}$ mutant, we hypothesized that MB neurons mediate ethanol-induced hyperactivity. To determine whether reduced tao function in the MB affects ethanolinduced hyperactivity, we tested the behavior of flies with strong tao RNAi knockdown driven by OK107-GAL4, which expresses in the MB but not the EB. MBspecific knockdown of tao reduced ethanolinduced hyperactivity (Fig. 6A,B). Notably, the ellipsoid body was left intact in these flies (Fig. 5F, G, I,J), implying that the effect of tao knockdown on ethanol-induced hyperactivity is not attributable to defects in the development of the EB. These experiments suggest that defects in $\mathrm{MB}$ morphology induced by tao knockdown are sufficient to produce a deficit in ethanolinduced hyperactivity.

To test the role of $\mathrm{MB}$ neurons in ethanol-induced hyperactivity directly, we performed a series of neuronal silencing experiments. We identified GAL4 lines that drive expression in $\mathrm{MB}$ neurons of all three classes, but not in EB neurons. Two such strains-OK107-GAL4 and 238YGAL4 - have been described previously (Aso et al., 2009). Two others-4.64GAL4 and 10.229-GAL4-were identified from a collection of P-GAL4 lines in a search for strains expressing in $\mathrm{MB}$ neurons. 4.64-GAL4 drives expression strongly in all three classes of $\mathrm{MB}$ neurons, as well as the

fan-shaped body and scattered neurons elsewhere in the brain and ventral nerve cord (VNC) (supplemental Fig. S4 A, B, available at www.jneurosci.org as supplemental material). 10.229-GAL4 drives expression predominantly in the $\mathrm{MB} \gamma$ neurons but also expresses in a subset of $\mathrm{MB} \alpha / \beta$ and $\alpha^{\prime} / \beta^{\prime}$ neurons (supplemental Fig. S4C,E, available at www.jneurosci.org as supplemental material). We observed no VNC expression with 10.229-GAL4 (supplemental Fig. S4D, available at www. jneurosci.org as supplemental material).

We used these GAL4 lines to express Kir2.1 to block neural activity (Baines et al., 2001). For strains that were lethal in combination with Kir2.1, we restricted expression to adult flies using the TARGET/Gal $80^{\text {ts }}$ system (McGuire et al., 2004). These flies were raised at a temperature permissive for Gal $80^{\text {ts }}$ repression, and then shifted to the restrictive temperature after eclosion to induce Kir2.1 expression. OK107-GAL4/+ controls failed to exhibit ethanol-induced hyperactivity under the temperature shift conditions needed to produce viable adults (data not shown), so it was excluded from our analysis. Expression of Kir2.1 in adult flies with 238Y-GAL4 resulted in gross locomotor defects even in the absence of ethanol (data not shown); this line was therefore also excluded. When 4.64-GAL4 and 10.229-GAL4 were combined with UAS-Kir2.1, they both reduced ethanol-induced hyperactivity compared with controls (Fig. 6C). These results are consistent with a role for the $\mathrm{MB}$ in the behavioral response to the stimulant effects of ethanol.
Control
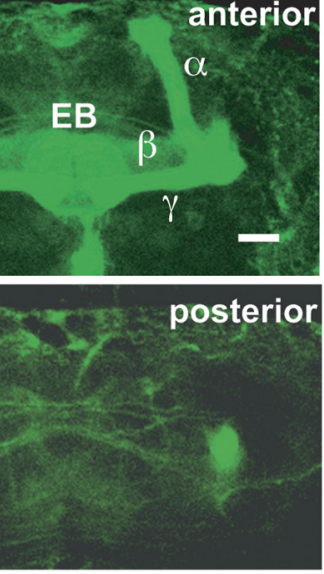
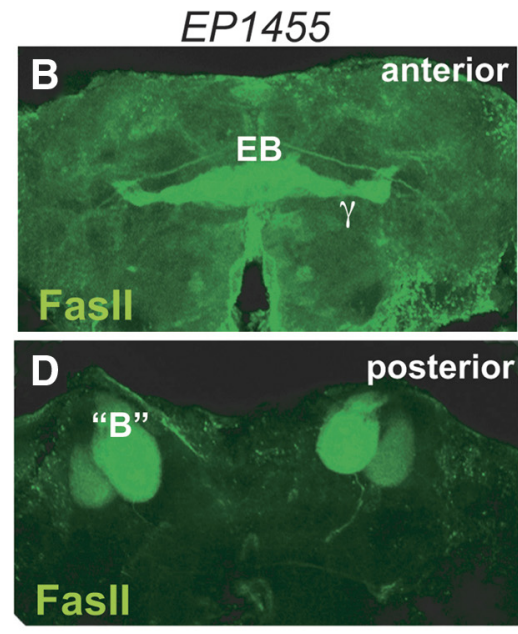

OK107-GAL4 + UAS-CD8.GFP
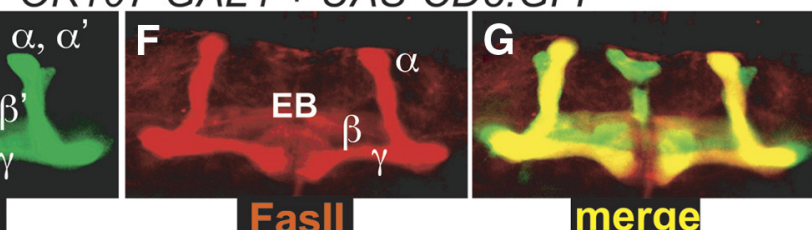

FasIl

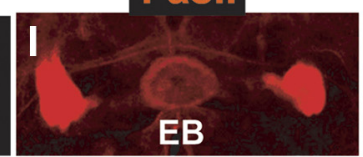

+ UAS-CD8.GFP

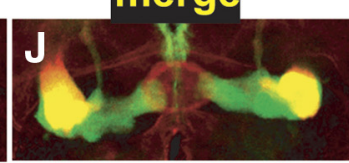

Figure 5. Defects in central brain morphology in tao mutants. $\boldsymbol{A}-\boldsymbol{D}$, Whole-mount brains from adult male wild-type $(\boldsymbol{A}$, $\boldsymbol{C})$ and $\operatorname{taO}^{E P 1455}(\boldsymbol{B}, \boldsymbol{D})$ flies were stained with anti-Fasciclin II, which marks the ellipsoid body (EB) and the $\alpha / \beta$ and $\gamma$ lobes dult male whole-mount brains with OK107-GAL4 driving expression of UAS-CD8.GFP (E-G) and UAS-tao ${ }^{\text {RNAi }, U A S-D C r 2, ~}$ and UAS-CD8.GFP $(\boldsymbol{H}-\boldsymbol{J})$. "B", Ball-like structure; P, peduncle. Scale bars, $15 \mu \mathrm{m}$. See also supplemental Figure S3 (available at www.jneurosci.org as supplemental material).

Because RNAi knockdown of tao induced its most severe defects in $\alpha / \beta$ neurons, we investigated whether silencing of $\alpha / \beta$ neurons alone was sufficient to affect ethanol-induced hyperactivity. We tested three P-GAL4 lines that drive expression in $\alpha / \beta$ neurons, but not in other MB neurons or the EB. $17 D$-GAL4 expresses in a specific subset of MB neurons (the $\alpha / \beta$ "core" and "surface" neurons) and has extremely limited expression elsewhere in the nervous system (Aso et al., 2009). When Kir2.1 was expressed using 17D-GAL4, ethanolinduced hyperactivity was reduced, suggesting that this subset of $\alpha / \beta$ neurons alone is sufficient to affect ethanol-induced hyperactivity (Fig. 6C). Like 17D-GAL4, the line NP6649GAL4 also drives expression in the $\alpha / \beta$ core/surface subset (Aso et al., 2009). NP6649-GAL4/+ controls had greatly reduced hyperactivity under the temperature shift regimen needed for adult expression of Kir2.1 (data not shown); we blocked synaptic transmission in these neurons by expressing tetanus toxin (TeTx) (Sweeney et al., 1995). NP6649-GAL4driven expression of UAS-TeTx also reduced ethanol-induced hyperactivity, further suggesting that this subset of $\alpha / \beta$ neurons is required for ethanol responsiveness (Fig. 6C). We also expressed UAS-TeTx with NP3208-GAL4, a line that expresses in a different subset of $\alpha / \beta$ neurons (the "posterior" subset) (Aso et al., 2009). This also resulted in reduced ethanolinduced hyperactivity (Fig. 6C), further demonstrating a role for $\alpha / \beta$ neurons in ethanol-stimulated behavior. 

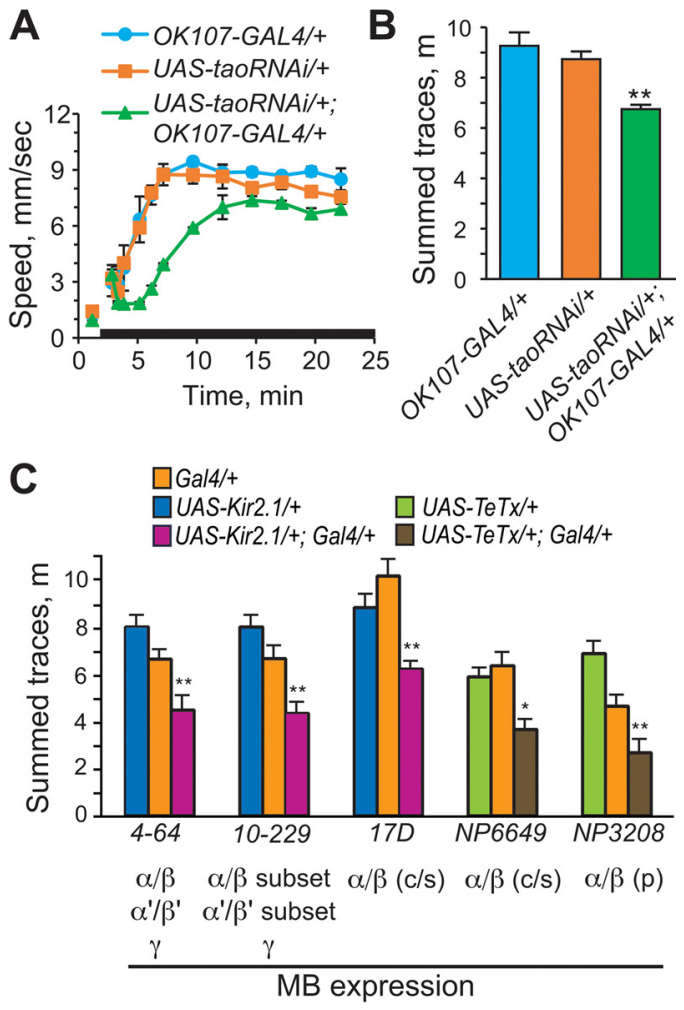

Figure 6. Silencing of $M B$ neurons reduces ethanol-induced hyperactivity. $A$, Ethanolinduced hyperactivity assay of flies with strong RNAi knockdown of tao expression in all MB neurons, as in Figure $5 E-J$. $\boldsymbol{B}$, Quantification of summed traces for $\boldsymbol{A}$. C, Quantification of summed traces from ethanol induced-hyperactivity assays, with Kir2.1 or TeTx silencing of MB neurons. The Gal $80^{\text {ts }} /$ TARGET system was used to restrict expression to adults for all driver lines except for 17D-GAL4 and NP6649-GAL4, which were viable with developmental expression of UAS-Kir2. 1 or UAS-TeTX. Flies were raised at $18^{\circ} \mathrm{C}$, and then shifted to $25^{\circ} \mathrm{C}$ for $2 \mathrm{~d}$ before testing. For experiments with $17 D-G A L 4$, flies were kept at $25^{\circ} \mathrm{C}$ throughout development and before testing. For experiments with NP6649-GAL4, flies were raised at $25^{\circ} \mathrm{C}$, and then shifted to $29^{\circ} \mathrm{C}$ for $2 \mathrm{~d}$ before testing. $\mathrm{c} / \mathrm{s}$, Core/surface; $\mathrm{p}$, posterior. Genotypes were compared by one-way ANOVA with Bonferroni's correction for multiple comparisons. ${ }^{*} p<0.05,{ }^{* *} p<0.01$. Error bars represent SEM. See also supplemental Figure $\$ 4$ (available at www.jneurosci.org as supplemental material).

\section{Mutations in par-1 suppress the developmental and behavioral phenotypes of tao mutations}

Previous studies suggest that par-1 is a likely signaling partner for tao in neural development. In mammals, TAO1/MARKK functions in the development of neurons in conjunction with the serine-threonine kinase Par-1/MARK2. In mammalian cells, TAO1 can phosphorylate and activate Par-1, which in turn can phosphorylate the microtubule-binding protein Tau, releasing it from microtubules (Biernat et al., 2002). In immature neurons, this results in an increase in microtubule dynamics, facilitating the outgrowth of neurites (Timm et al., 2003). However, in Drosophila, Tao overexpression reduces phosphorylation of Par-1 at the activating site T408, suggesting a negative relationship between tao and par-1 in flies (Wang et al., 2007).

We investigated the relationship between tao and par-1 in our system by testing whether mutations in par-1 affected the morphological and behavioral phenotypes of tao mutations. We first examined the MB morphology of flies carrying both $\mathrm{tao}^{E P 1455}$ and loss-of-function alleles of par-1. Two strong loss-of-function alleles, par- $1^{\text {W3 }}$ (Shulman et al., 2000) and par- $1^{k 05603}$ (Tomancak et al., 2000), both suppressed the MB morphology defect of tao ${ }^{E P 1455}$ as heterozygotes (Fig. $7 A-F$ ). $t_{a 0}{ }^{E P 1455}$ flies rarely had fully formed $\mathrm{MB} \alpha$ or $\beta$ lobes, but double mutants with either par-1 allele had fully formed lobes in approximately one-half of all brain hemispheres (Fig. 7A-F; supplemental Fig. S5A, available at www.jneurosci.org as supplemental material). To determine whether the suppression of tao morphology phenotypes was attributable to interactions between tao and par-1 autonomous to the $\mathrm{MB}$, we tested whether par-1 mutations could also suppress a MB-specific tao RNAi knockdown phenotype. Weak RNAi knockdown in the MB produces a moderate MB morphology phenotype, which is much less severe than that of tao ${ }^{E P 1455}$ (Fig. 7G; supplemental Fig. S5B, available at www.jneurosci.org as supplemental material). Heterozygosity of par- $1^{W 3}$ suppressed the tao RNAi phenotype considerably (Fig. $7 H$; supplemental Fig. $\mathrm{S} 5 B$, available at www.jneurosci.org as supplemental material), indicating that the interaction between tao and par-1 mutations is $\mathrm{MB}$ autonomous. These results are consistent with tao acting as a negative upstream regulator of par-1 (Wang et al., 2007) and raises the possibility that the functional relationship between these genes in Drosophila is different from that described in mammalian cells.

Because par-1 mutations can suppress tao phenotypes, the phenotype of tao mutants might be attributable to an increase in par-1 activity in developing MB neurons. To test whether increased par-1 activity could affect MB development, we overexpressed Par-1 with OK107-GAL4 and examined MB morphology with FasII staining. Overexpression of Par-1 disrupted the formation of $\mathrm{MB} \alpha / \beta$ lobes, similar to the defect seen in tao mutants (Fig. $7 I, J)$. Abnormal $\beta$ lobes were more common than disrupted $\alpha$ lobes, although Par-1 overexpression affected both (supplemental Fig. S5C, available at www.jneurosci.org as supplemental material). We also overexpressed Par- ${ }^{\mathrm{T} 408 \mathrm{~A}}$, in which the activating phosphorylation site T408 has been mutated to alanine. Overexpression of Par- ${ }^{\text {T408A }}$ with OK107-GAL4 did not affect MB morphology (Fig. $7 K, L$ ). This suggests that phosphorylation of T408, which can be regulated by Tao overexpression, is critical to $\mathrm{MB}$ development.

Par-1 overexpression in the eye has been shown to disrupt the development of photoreceptor neurons and can contribute to Tau-mediated neurodegeneration (Nishimura et al., 2004). Par-1 phosphorylates Tau directly at residues S262 and S356, enabling additional phosphorylation that contributes to neurodegeneration (Nishimura et al., 2004). We tested whether Tau phosphorylation by Par-1 in developing neurons was affected by knockdown of tao. Par-1 was overexpressed in the eye with or without UAS-tao ${ }^{R N A i}$, and phosphorylation of Tau at S262 and S356 measured by Western blotting with the monoclonal antibody $12 \mathrm{E} 8$, which specifically recognizes Tau phosphorylated at these sites. Coexpression of Par-1 and tao RNAi lead to an increase in phosphorylation of Tau compared with expression of Par-1 alone (Fig. 7M), consistent with regulation of Par-1 by Tao. Tau expression levels were not affected by tao RNAi, as seen by blotting with the monoclonal antibody T14 (Fig. 7M). These experiments suggest that Tao acts in a pathway with Par-1 and Tau during MB development.

We also tested whether loss of par-1 function could suppress the behavioral phenotype of tao ${ }^{E P 1455}$. Double mutants hemizygous for $t a o^{E P 1455}$ and heterozygous for par-1 ${ }^{k 05603}$ were compared with single mutant controls for ethanol-induced hyperactivity. par- $1^{k 05603} /+$ heterozygotes had normal ethanolinduced hyperactivity and tao ${ }^{E P 1455}$ hemizygotes showed their typical reduced hyperactivity; in the double mutant, however, the tao ${ }^{E P 1455}$ phenotype was strongly suppressed (Fig. $7 \mathrm{~N}, \mathrm{O}$ ). Thus, the suppression of the MB morphology phenotype of tao ${ }^{E P 1455}$ correlated well with the suppression of its behavioral phenotype, 
providing additional evidence that the defects in brain morphology observed in tao mutants are responsible for their behavioral phenotypes.

\section{Discussion}

We have identified a mutant in the Drosophila Ste20 family serine-threonine kinase tao that has profound effects on ethanol-induced locomotor hyperactivity, a behavior associated with the disinhibiting and rewarding effects of ethanol in mammals. tao mutants affect multiple drug responses, also causing resistance to the effects of cocaine and nicotine. Furthermore, we have identified the $\mathrm{MB}$ as part of the neural circuitry that mediates ethanol-induced hyperactivity. We have determined that tao function is required for the proper development of the adult nervous system, particularly the $\mathrm{MB}$, and that this developmental function is needed for normal ethanol-stimulated activity. By selectively silencing $\mathrm{MB}$ neurons, we have shown that the $\mathrm{MB} \alpha / \beta$ neurons are required for normal behavior. Finally, we have determined that tao acts as a negative regulator of par-1 and that the phenotypes of tao mutants are likely a result of effects on phosphorylation of the microtubule-binding protein Tau. We show that par-1 mutations suppress tao mutant phenotypes and that tao mutations can affect the phosphorylation of Tau by Par-1. Thus, tao likely exerts its effects on $\mathrm{MB}$ development and ethanolinduced hyperactivity as part of a pathway that regulates microtubule dynamics.

\section{Mushroom body in drug response \\ behavior}

Our study points to the $\mathrm{MB}$, and particularly the $\alpha / \beta \mathrm{MB}$ neurons, as important mediators of ethanol-induced hyperactivity, a function not previously attributed to these neurons. We have also observed that ethanol-induced hyperactivity is affected by the electrical and synaptic silencing of two non-overlapping subsets of $\alpha / \beta$ neurons. NP6649-GAL4 and 17D-GAL4 both express in only the core and surface $\alpha / \beta$ neurons, whereas NP3208-GAL4 expresses in a separate posterior subset (Aso et al., 2009). Our results suggest that, for normal ethanol-induced hyperactivity, these different subsets of $\alpha / \beta$ neurons may be functionally equivalent.

Although defects in the formation of the $\mathrm{MB}$ contribute to the phenotype of tao mutants, the mutant phenotype of tao ${ }^{E P 1455}$ is much more severe than the phenotypes caused by the silencing of MB neurons. This suggests that Tao is needed for the normal development of additional brain regions. For example, the EB morphology defects found in $\mathrm{tao}^{E P 1455}$ flies might also contribute to their behavioral phenotype. A detailed study of EB neurons has revealed that synaptic silencing of the $\mathrm{R} 2$ and $\mathrm{R} 4$ neurons of the EB impairs ethanol-induced hyperactivity (Kong et al., 2010).

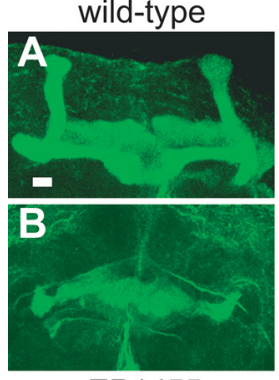

EP1455
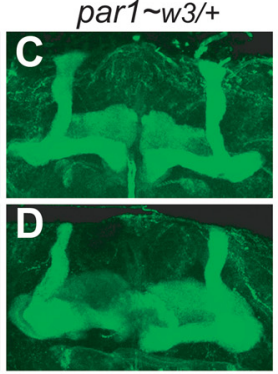

EP1455; par1 w3/+
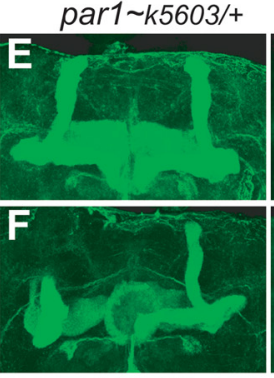

EP1455;

par1 k5603/+

OK107-GAL4/+; UAS-tao.RNAi/+

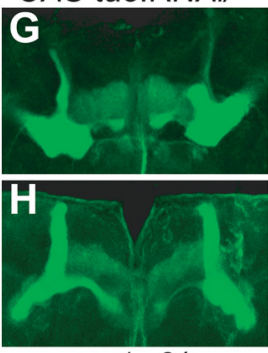

par1w3/+

OK107-GAL4/+

UAS-tao.RNAi/+
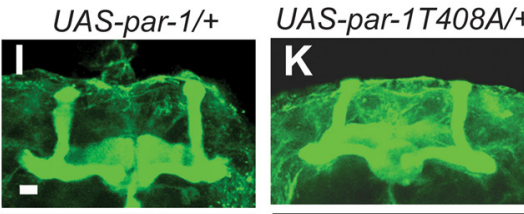

M

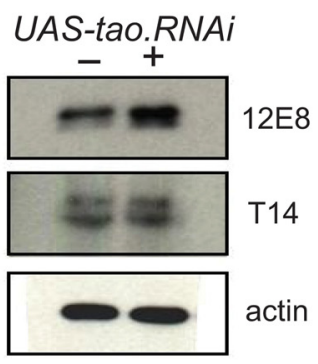

GMR-Gal4/+;UAS-par-1/+

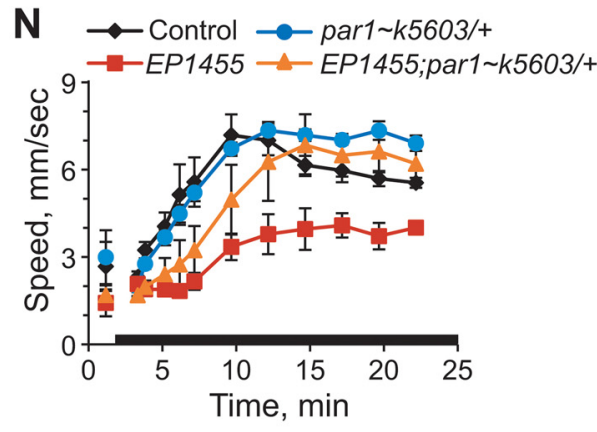

Figure 7. Mutations in par-1 suppress tao phenotypes. $\boldsymbol{A}-\boldsymbol{H}$, Anterior projections of adult male whole-mount brains stained

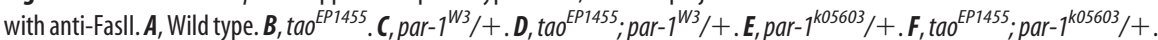

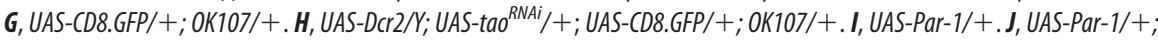

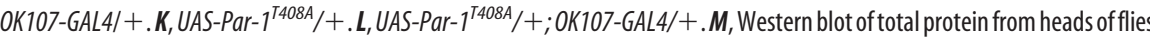
verexpressing Par-1 with GMR-GAL4 with and without UAS-tao ${ }^{R N A i}$, probed with antibodies to Tau (T14), phospho-Tau (12E8), $7^{k 05603} /+$. O, Quantification of summed traces from $N$. Genotypes were compared by one-way ANOVA with Bonferroni's correction for multiple comparisons. ${ }^{* *} p<0.01$. Error bars represent SEM. Scale bar, $15 \mu \mathrm{m}$. See also supplemental Figure S5 (available at www.jneurosci.org as supplemental material).

Consistent with this, expression of tao with 5.30-GAL4, which expresses in EB R2/R4 neurons, is able to partially rescue the phenotype of $t_{a o^{E P 1455}}$ (data not shown). It is likely that there are also other as-yet-unidentified brain regions affected by tao mutations that contribute to their drug response phenotypes.

Additional studies are needed to elucidate whether different ethanol-induced behaviors in Drosophila have common underlying circuitry. Mutations in the neuropeptide-encoding gene amnesiac, which is highly expressed in a pair of neurons that broadly innervate the MB lobes (Waddell et al., 2000), strongly affect ethanol-induced loss of postural control (Moore et al., 1998), suggesting the MB may indeed influence additional ethanol- 
induced behaviors. However, amnesiac is also expressed elsewhere in the fly, and its spatial requirement for ethanol sensitivity has not been mapped. Moreover, chemical ablation of MB neurons does not affect ethanol-induced sedation (Rodan et al., 2002). Additional studies are therefore needed to define the circuitry required for these two additional ethanol-induced behaviors.

Since tao mutants are resistant to the effects of nicotine and cocaine as well as to the stimulant effects of ethanol, it is possible that the MB might also be required for behaviors induced by other drugs of abuse. Like mutations in tao, mutations in the gene white rabbit (whir) affect responses to cocaine, nicotine, and ethanol-induced sedation and hyperactivity (Rothenfluh et al., 2006). The ethanol-induced sedation phenotype of whir can be rescued with expression driven by a whir enhancer trap line whose expression pattern includes $\mathrm{MB} \alpha / \beta$ neurons (Rothenfluh et al., 2006), raising the possibility that function of whir in the MB might be at least partially responsible for its multiple drug response phenotypes. Additional analysis of tao and whir mutants, and the neurons in which these genes function, will help identify circuitry common or unique to the behavioral responses to different drugs of abuse in Drosophila.

\section{tao in neuronal development}

The phenotype of tao mutants suggests that tao functions in axon guidance in the developing MB. tao ${ }^{E P 1455}$ flies lack MB $\alpha / \beta$ lobes, and anti-FasII staining reveals large ball-like formations just below the MB calices. This phenotype is very similar to defects observed in loss-of-function mutations in the Rac family of small GTPases Rac1, Rac2, and Mtl: flies mutant for all three Rac genes display the same ball-like cluster of FasII staining below the calices (Ng et al., 2002). Clonal analysis revealed that the Rac phenotype results from a defect in axon guidance, as the axons of developing $\mathrm{MB}$ neurons are able to extend but aggregate into a ball rather than forming axon tracts (Ng et al., 2002).

Rac family genes and other genes involved in axon guidance of $\mathrm{MB}$ neurons often show defects in multiple aspects of neuronal development, including cell proliferation and/or survival, axon extension and branching, and dendrite morphogenesis ( $\mathrm{Ng}$ et al., 2002; Reuter et al., 2003). tao mutations do not have obvious effects on other aspects of MB development: Kenyon cell number and the calyx (containing Kenyon cell dendrites) appear normal (data not shown). Although a more careful analysis will be necessary to determine whether tao is involved in other aspects of $\mathrm{MB}$ morphogenesis, our analysis suggests that in $\mathrm{MB}$ neurons tao mutations primarily affect axon guidance.

The regulatory relationship between tao and par-1 appears to be reversed in mammalian cells and Drosophila. In cultured mammalian cells, neurite outgrowth can be regulated by TAO1 activation of Par-1 (Biernat et al., 2002; Timm et al., 2003). However, we have shown that loss-of-function mutations in par-1 suppress tao mutant phenotypes, implying negative regulation of Par-1 by Tao. Although it is not known whether the effects that we observe on $\mathrm{MB}$ morphogenesis involve a physical interaction between Tao and Par-1 as is seen in mammalian cells, the MB development phenotype seen with Par-1 overexpression is dependent on T408, a Tao phosphorylation site (Wang et al., 2007), suggesting a direct interaction. This difference in the pathways by which TAO family kinases control neural development in flies and mammals might be attributable to the action of upstream factors or might reflect a fundamental difference in the function of these proteins in flies and mammals.

We have observed that the behavioral phenotype of $\operatorname{tao}^{E P 1455}$ is temperature sensitive; the mutant displays normal ethanol- induced hyperactivity behavior when kept at the permissive temperature of $18^{\circ} \mathrm{C}$ during metamorphosis but has reduced hyperactivity when kept at the restrictive temperature of $25^{\circ} \mathrm{C}$. The reason for the temperature sensitivity of the phenotype is unknown and could result from a number of causes. Because tao ${ }^{E P 1455}$ carries a P-element insertion in the $5^{\prime}$-UTR of the gene, the coding sequence of tao is not altered in the mutant, and the temperature sensitivity of the allele is therefore not attributable to changes in the stability of the protein product at different temperatures. However, we have observed that splicing of the tao gene is altered by the presence of the transposon; using reverse transcription-PCR, we have observed that in $t a o^{E P 1455}$ mRNA products containing transposon sequences are transcribed and that splicing occurs from within the transposon sequences to the second tao exon, eliminating some of the $5^{\prime}$-UTR and replacing it with transposon sequences (data not shown). It is possible that this aberrant splicing pattern affects splicing kinetics, mRNA stability, or translation in a temperature-sensitive manner, making the mutant phenotype temperature sensitive as well. Finally, it is possible that the cell biological process in which Tao functions becomes temperature sensitive when Tao levels are limiting.

Tao might have a number of functions in the nervous system and elsewhere, and might be used in multiple signaling cascades. For instance, mammalian TAO kinases have also been identified as upstream activators of 338 MAPK (mitogen-activated protein kinase) signaling in cellular reactions to stress and might activate this pathway in flies (Chen et al., 1999, 2003; Raman et al., 2007; Yasuda et al., 2007). TAO-2 also activates p38 signaling as part of a pathway controlling dendritic spine plasticity in the mammalian brain, another potential function of tao in the Drosophila nervous system (Yasuda et al., 2007). These and other molecular mechanisms might also in part underlie the behavioral phenotypes of tao mutants.

\section{References}

Aso Y, Grübel K, Busch S, Friedrich AB, Siwanowicz I, Tanimoto H (2009) The mushroom body of adult Drosophila characterized by GAL4 drivers. J Neurogenet 23:156-172.

Baines RA, Uhler JP, Thompson A, Sweeney ST, Bate M (2001) Altered electrical properties in Drosophila neurons developing without synaptic transmission. J Neurosci 21:1523-1531.

Bainton RJ, Tsai LT, Singh CM, Moore MS, Neckameyer WS, Heberlein U (2000) Dopamine modulates acute responses to cocaine, nicotine and ethanol in Drosophila. Curr Biol 10:187-194.

Biernat J, Wu YZ, Timm T, Zheng-Fischhöfer Q, Mandelkow E, Meijer L, Mandelkow EM (2002) Protein kinase MARK/PAR-1 is required for neurite outgrowth and establishment of neuronal polarity. Mol Biol Cell 13:4013-4028.

Bland ND, Robinson P, Thomas JE, Shirras AD, Turner AJ, Isaac RE (2009) Locomotor and geotactic behavior of Drosophila melanogaster overexpressing neprilysin 2. Peptides 30:571-574.

Chen Z, Hutchison M, Cobb MH (1999) Isolation of the protein kinase TAO2 and identification of its mitogen-activated protein kinase/extracellular signal-regulated kinase kinase binding domain. J Biol Chem 274:28803-28807.

Chen Z, Raman M, Chen L, Lee SF, Gilman AG, Cobb MH (2003) TAO (thousand-and-one amino acid) protein kinases mediate signaling from carbachol to p38 mitogen-activated protein kinase and ternary complex factors. J Biol Chem 278:22278-22283.

Dan I, Watanabe NM, Kusumi A (2001) The Ste20 group kinases as regulators of MAP kinase cascades. Trends Cell Biol 11:220-230.

Devineni AV, Heberlein U (2009) Preferential ethanol consumption in Drosophila models features of addiction. Curr Biol 19:2126-2132.

Guarnieri DJ, Heberlein U (2003) Drosophila melanogaster, a genetic model system for alcohol research. Int Rev Neurobiol 54:199-228.

Hoskins RA, Nelson CR, Berman BP, Laverty TR, George RA, Ciesiolka L, Naeemuddin M, Arenson AD, Durbin J, David RG, Tabor PE, Bailey MR, 
DeShazo DR, Catanese J, Mammoser A, Osoegawa K, de Jong PJ, Celniker SE, Gibbs RA, Rubin GM, et al. (2000) A BAC-based physical map of the major autosomes of Drosophila melanogaster. Science 287:2271-2274.

Kong EC, Woo K, Li H, Lebestky T, Mayer N, Sniffen MR, Heberlein U, Bainton RJ, Hirsh J, Wolf FW (2010) A pair of dopamine neurons target the D1-like dopamine receptor DopR in the central complex to promote ethanol-stimulated locomotion in Drosophila. PLoS One 5:e9954.

Lee T, Lee A, Luo L (1999) Development of the Drosophila mushroom bodies: sequential generation of three distinct types of neurons from a neuroblast. Development 126:4065-4076.

Lin DM, Goodman CS (1994) Ectopic and increased expression of Fasciclin II alters motoneuron growth cone guidance. Neuron 13:507-523.

McGuire SE, Mao Z, Davis RL (2004) Spatiotemporal gene expression targeting with the TARGET and gene-switch systems in Drosophila. Sci STKE 2004:pl6.

Moore MS, DeZazzo J, Luk AY, Tully T, Singh CM, Heberlein U (1998) Ethanol intoxication in Drosophila: genetic and pharmacological evidence for regulation by the cAMP signaling pathway. Cell 93:997-1007.

Ng J, Nardine T, Harms M, Tzu J, Goldstein A, Sun Y, Dietzl G, Dickson BJ, Luo L (2002) Rac GTPases control axon growth, guidance and branching. Nature 416:442-447.

Nishimura I, Yang Y, Lu B (2004) PAR-1 kinase plays an initiator role in a temporally ordered phosphorylation process that confers tau toxicity in Drosophila. Cell 116:671-682.

Phillips TJ, Shen EH (1996) Neurochemical bases of locomotion and ethanol stimulant effects. Int Rev Neurobiol 39:243-282.

Raman M, Earnest S, Zhang K, Zhao Y, Cobb MH (2007) TAO kinases mediate activation of $\mathrm{p} 38$ in response to DNA damage. EMBO J 26:2005-2014.

Reuter JE, Nardine TM, Penton A, Billuart P, Scott EK, Usui T, Uemura T, Luo L (2003) A mosaic genetic screen for genes necessary for Drosophila mushroom body neuronal morphogenesis. Development 130:12031213.

Rodan AR, Kiger JA Jr, Heberlein U (2002) Functional dissection of neuroanatomical loci regulating ethanol sensitivity in Drosophila. J Neurosci 22:9490-9501.

Rørth P (1996) A modular misexpression screen in Drosophila detecting tissue-specific phenotypes. Proc Natl Acad Sci U S A 93:12418-12422.

Rothenfluh A, Threlkeld RJ, Bainton RJ, Tsai LT, Lasek AW, Heberlein U (2006) Distinct behavioral responses to ethanol are regulated by alternate RhoGAP18B isoforms. Cell 127:199-211.

Rubin GM, Spradling AC (1982) Genetic transformation of Drosophila with transposable element vectors. Science 218:348-353.

Sato K, Hayashi Y, Ninomiya Y, Shigenobu S, Arita K, Mukai M, Kobayashi S
(2007) Maternal Nanos represses hid/skl-dependent apoptosis to maintain the germ line in Drosophila embryos. Proc Natl Acad Sci U S A 104:7455-7460.

Scholz H, Ramond J, Singh CM, Heberlein U (2000) Functional ethanol tolerance in Drosophila. Neuron 28:261-271.

Sharma Y, Cheung U, Larsen EW, Eberl DF (2002) PPTGAL, a convenient Gal4 P-element vector for testing expression of enhancer fragments in Drosophila. Genesis 34:115-118.

Shulman JM, Feany MB (2003) Genetic modifiers of tauopathy in Drosophila. Genetics 165:1233-1242.

Shulman JM, Benton R, St Johnston D (2000) The Drosophila homolog of C. elegans PAR-1 organizes the oocyte cytoskeleton and directs oskar mRNA localization to the posterior pole. Cell 101:377-388.

Sweeney ST, Broadie K, Keane J, Niemann H, O’Kane CJ (1995) Targeted expression of tetanus toxin light chain in Drosophila specifically eliminates synaptic transmission and causes behavioral defects. Neuron 14:341-351.

Timm T, Li XY, Biernat J, Jiao J, Mandelkow E, Vandekerckhove J, Mandelkow EM (2003) MARKK, a Ste20-like kinase, activates the polarityinducing kinase MARK/PAR-1. EMBO J 22:5090-5101.

Timm T, Matenia D, Li XY, Griesshaber B, Mandelkow EM (2006) Signaling from MARK to tau: regulation, cytoskeletal crosstalk, and pathological phosphorylation. Neurodegener Dis 3:207-217.

Tomancak P, Piano F, Riechmann V, Gunsalus KC, Kemphues KJ, Ephrussi A (2000) A Drosophila melanogaster homologue of Caenorhabditis elegans par-1 acts at an early step in embryonic-axis formation. Nat Cell Biol 2:458-460.

Truman JW (1990) Metamorphosis of the central nervous system of Drosophila. J Neurobiol 21:1072-1084.

Waddell S, Armstrong JD, Kitamoto T, Kaiser K, Quinn WG (2000) The amnesiac gene product is expressed in two neurons in the Drosophila brain that are critical for memory. Cell 103:805-813.

Wang JW, Imai Y, Lu B (2007) Activation of PAR-1 kinase and stimulation of tau phosphorylation by diverse signals require the tumor suppressor protein LKB1. J Neurosci 27:574-581.

Wolf FW, Rodan AR, Tsai LT, Heberlein U (2002) High-resolution analysis of ethanol-induced locomotor stimulation in Drosophila. J Neurosci 22:11035-11044.

Yasuda S, Tanaka H, Sugiura H, Okamura K, Sakaguchi T, Tran U, Takemiya T, Mizoguchi A, Yagita Y, Sakurai T, De Robertis EM, Yamagata K (2007) Activity-induced protocadherin arcadlin regulates dendritic spine number by triggering N-cadherin endocytosis via TAO2beta and p38 MAP kinases. Neuron 56:456-471. 